\title{
Renato Pasquali
}

\author{
Uberto Pagotto ${ }^{1}$. Valentina Vicennati ${ }^{1}$. Danilo Ribichini ${ }^{1}$. Carla Pelusi ${ }^{1}$ - Paola Altieri ${ }^{1}$. Silvia Garelli ${ }^{1}$. \\ Andrea Repaci ${ }^{1}$. Flaminia Fanelli ${ }^{1}$. Guido Di Dalmazi ${ }^{1}$. Alessandra Gambineri ${ }^{1}$
}

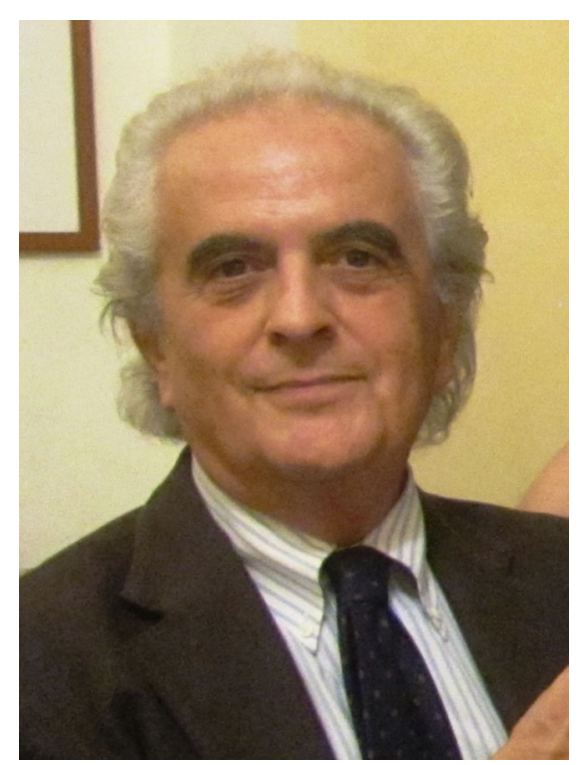

L'immagine, forte e netta, che tratteniamo del Professor Renato Pasquali (Budrio, 1946-Bologna, 2019) dopo molti e intensi anni di collaborazione, è prima di tutto quella dell'hombre vertical, dell'uomo e del Professore tutto d'un pezzo, una roccia di valori morali.

Il Professore Renato Pasquali, il nostro Maestro, ha creato l'Endocrinologia Universitaria di Bologna partendo dal nulla, dopo aver lavorato nell'Istituto di Clinica Medica sotto l'illuminata direzione dei Professori Labò e Barbara. Di quegli anni, che ricordava sempre con enorme trasporto e piacere, aveva derivato la consapevolezza che la medi-

U. Pagotto

uberto.pagotto@unibo.it

1 U.O.C. Endocrinologia e Prevenzione e Cura del Diabete, Policlinico di S. Orsola, Università Alma Mater Studiorum di Bologna, Bologna, Italia cina è prima di tutto metodo e studio, ma anche passione e compassione. Da questo ambiente fecondo è sorta la sua passione per le malattie metaboliche, sulle quali al principio collaborò con il Professor Melchionda, per poi iniziare, con il supporto del Professor Barbara, una carriera indipendente da endocrinologo che lo ha portato a fondare nel 1998 l'Unità Operativa di Endocrinologia del Policlinico S. OrsolaMalpighi da lui diretta fino al 2016, al termine della sua carriera universitaria (Fig. 1).

Il suo impegno costante per i valori e per le persone si è riflesso nella grande crescita clinica e scientifica dell'Endocrinologia bolognese che si è affermata a livello nazionale $\mathrm{e}$ internazionale sotto la sua guida. Era un uomo di grandi visioni, spesso provocatorie, ma di illuminante effetto; visioni sempre finalizzate ad affrontare i problemi, mai a eluderli. Era un uomo di grande e plastica cultura non esclusivamente medica, i suoi interessi andavano dalla filosofia alla storia, dall'arte al football, ma soprattutto la sua passione era rivolta al malato come un unicum, pur nella sua complessità. È stato per tutti i suoi allievi un grande Maestro e un sicuro punto di riferimento prima di tutto per due caratteristiche che risaltavano come fari nelle nebbie: la saldezza morale e la metodologia clinica come disciplina primaria nella pratica medica. È stato anche un Maestro talvolta duro, poco incline a svenevolezze e sotterfugi; amava la disciplina e il ruolo gerarchico, da vero accademico d'antan, ma era comunque un uomo pieno di slanci e di reale affetto nei confronti del suo gruppo, gruppo che riconosceva, a sua volta, la sua immensa bravura e al tempo stesso amava i suoi estri.

La sua orgogliosa spinta a fondare la Scuola endocrinologica universitaria bolognese ha permeato ogni istante della sua vita clinica e accademica. Tale spinta è stata messa, talvolta, a dura prova dagli avvenimenti esterni, ma la sua incrollabile fede nella forza del lavoro ben fatto, nella rigorosa applicazione del metodo clinico-scientifico, fosse 


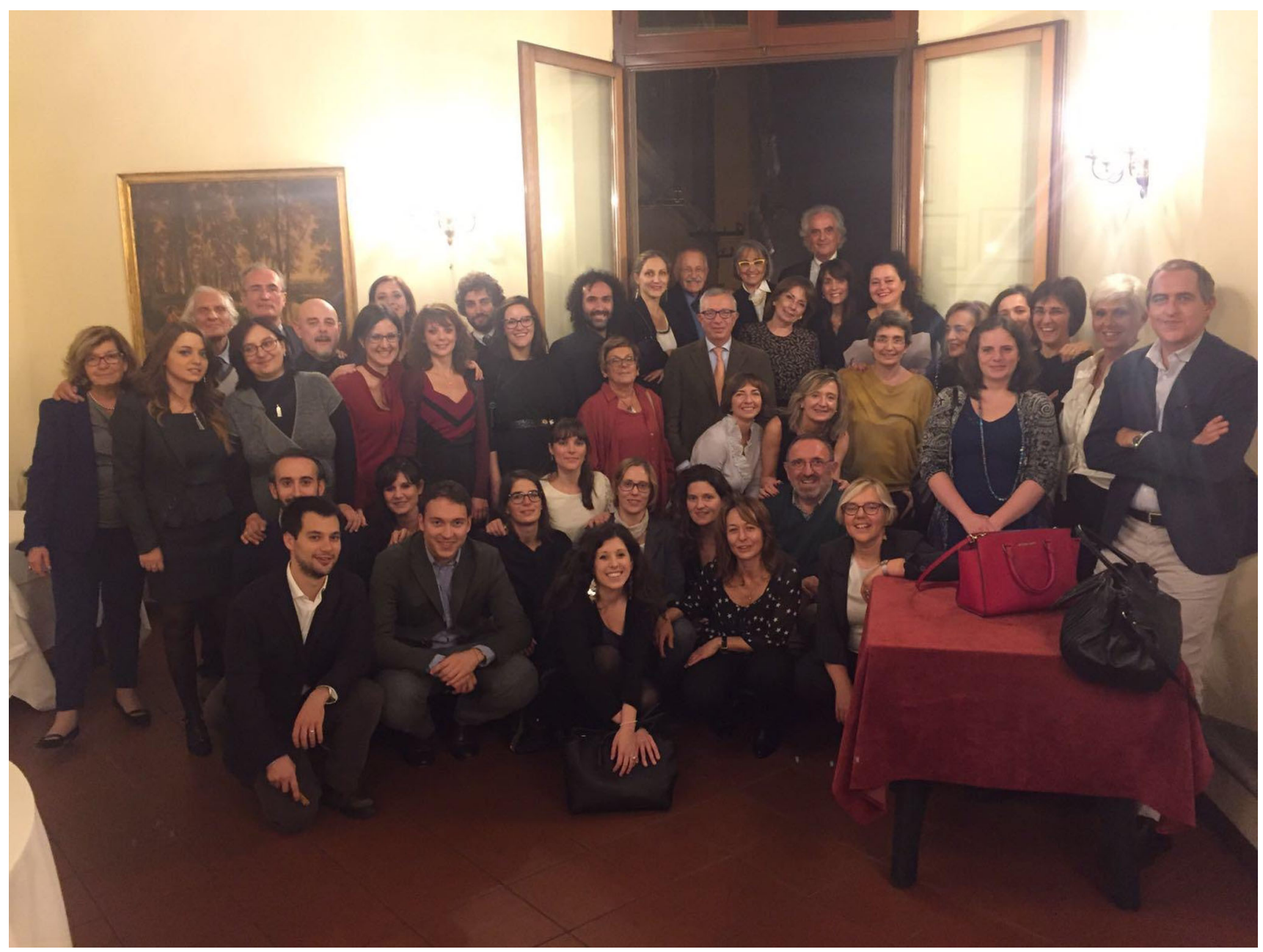

Fig. 1 Il Professor Renato Pasquali con la sua Scuola di ieri e di oggi e alcuni colleghi bolognesi alla festa (a sorpresa) in occasione del suo ritiro dall' attività accademica

per una diagnosi come per una pubblicazione, e nell'ostinata volontà di credere sempre a un sogno, lo ha nell'ultimo periodo della carriera reso felicemente consapevole che la sua Scuola Endocrinologica Universitaria bolognese era una realtà inscalfibile.

Godeva di una grande stima nell'ambito delle Società Scientifiche che frequentava con grande piacere. $\mathrm{Ci}$ piace ricordare, perché vero e molto da noi apprezzato, quanto scrittoci alla notizia della sua morte da un collega straniero che ha con lui collaborato per anni. Il collega, ricordando il Professor Pasquali, lo ha dipinto come un uomo del Rinascimento, lasciando così intendere lo stile, l'eleganza, la maestria, la bravura e la cultura, tipica di quel periodo storico tutto italiano, del nostro Maestro. Qui in Italia e molto anche fuori dai confini del nostro paese, siamo sicuri, la sua tragica scomparsa ha lasciato attoniti e increduli, un folto gruppo di endocrinologi e di medici di altre discipline, colleghi che lui ha sempre, e fortemente, considerato come veri amici.
Renato Pasquali ha pubblicato più di 300 lavori su riviste internazionali, è stato ispiratore di linee guida mondiali di patologie quali la sindrome dell'ovaio policistico (il suo grande cavallo di battaglia) e l'obesità e, quale riconoscimento per la sua importante attività scientifica, ha ricevuto due anni fa a Lisbona il prestigiosissimo premio europeo alla carriera da parte della European Society of Endocrinology. Ricordiamo ancora la sua fortissima emozione sul palco per il premio a Lisbona, premio che lui per primo considerava meritato, perché assegnato secondo le regole della solida scienza che lui aveva nella sua carriera sempre rispettato. Qui però, oltre ai lavori e ai riconoscimenti, va ricordata la sua magistrale capacità di cucire solidissime traiettorie tra argomenti endocrinologici apparentemente distanti, perfettamente rappresentate dai suoi molto citati studi sugli steroidi nelle loro varie espressioni in differenti patologie endocrino-metaboliche. Ha servito la Società Italiana di Endocrinologia come membro di varie Commissioni e del Direttivo ed è stato per molti anni nell'Editorial Board del Journal of Endocrinological Investigation. Diede anche un forte 
e ineguagliabile contributo alla rivista L'Endocrinologo per la quale generò, d'intesa con il Professor Francesco Trimarchi, la rubrica "Pratica clinica in endocrinologia". Anche dopo il termine della sua carriera accademica, ha continuato il suo contributo scientifico con la stesura e la recente pubblicazione delle linee guida europee sull'obesità sullo European Journal of Endocrinology; inoltre, proprio in questi giorni stava ultimando una review sul ruolo degli androgeni nella donna per un prestigioso giornale internazionale di
Endocrinologia.

Noi che siamo tenuti a portare avanti il suo sogno con le medesime regole di passione, di culto del lavoro e di intransigenza che ci sono state da lui insegnate, nella nostra grande tristezza, siamo fortemente orgogliosi di averlo avuto come Maestro e di averlo aiutato a fondare la Unità Operativa di Endocrinologia del Policlinico S. Orsola dell'Alma Mater Studiorum Università di Bologna. 